\title{
Benign Neonatal Sleep Myoclonus (BNSM) : A Case Report
}

\author{
KHAJA SULTAN ALI ${ }^{1}$, MAY MAY HAL MARMA ${ }^{2}$
}

\section{Introduction}

Neonatal sleep myoclonus is a benign disorder characterized by myoclonic jerks occurring only during sleep and presenting in the first month of life. The condition is self-limited, requires no treatment and is unassociated with any developmental and neurological deficits. ${ }^{1,2}$ The fact that benign neonatal sleep myoclonus (BNSM) is an innocuous and non-epileptic phenomenon is not well recognized. ${ }^{2,3}$ Not uncommonly, BNSM is confused with neonatal seizure disorder, resulting in unnecessary investigations, treatment and parental anxiety. ${ }^{1,2}$

\section{Case report}

A 29-day-male, weighing $3 \mathrm{~kg}$, exclusive breast fed infant presented with multiple episodes of abnormal jerks affecting all the four limbs, not associated with any change in color. In between the episodes he was active, and feeding well. This baby was born at a local hospital following a term gestation, to a primigravida mother. Baby had cried immediately at birth on the same day he was admitted in Shahbuddin Medical College Hospital neonatal intensive care unit on 26th January 2014, due to neonatal sepsis, He was treated accordingly and discharged after 7 days. Again he was brought on day 29 with a history suggestive of abnormal movements of limbs noted for 14 days. There was no other significant contributory history and the family history was negative for any seizure disorder. The baby was admitted with a diagnosis of neonatal seizures.

On admission baby was awake, alert, had no neurological deficits. In the hospital, the jerky movements lasted between 30 seconds to 2 minutes, mostly involving distal part of limbs without any change in vital parameters. Blood glucose, calcium, and septic work-up were all normal. Treatment with oral phenobarbitone, did not prevent recurrences of myoclonic jerks. MRI brain and sleep EEG record (Fig.-1) did not reveal any abnormality. Possibility of BNSM was considered. Review of history with mother

1. Associate Professor of Pediatrics, Shahabuddin Medical College, Gulshan, Dhaka.

2. Registrar of Pediatrics, Shahabuddin Medical College, Gulshan, Dhaka.

Correspondence: Dr. Khaja Sultan Ali Ahmed. E-mail: khajasultan@hotmail.com revealed that jerks occurred during sleep, repetitive head-to-toe rocking, travelling by auto rickshaw or cycle rickshaw and increase during holding of the baby to stop jerky movement. Myoclonic movements disappear as soon as the baby awake. An observation confirmed during the hospital stay of the baby. Anticonvulsants were tapered gradually. Baby was under follow up. Myoclonus disappeared completely by 4 months of age.

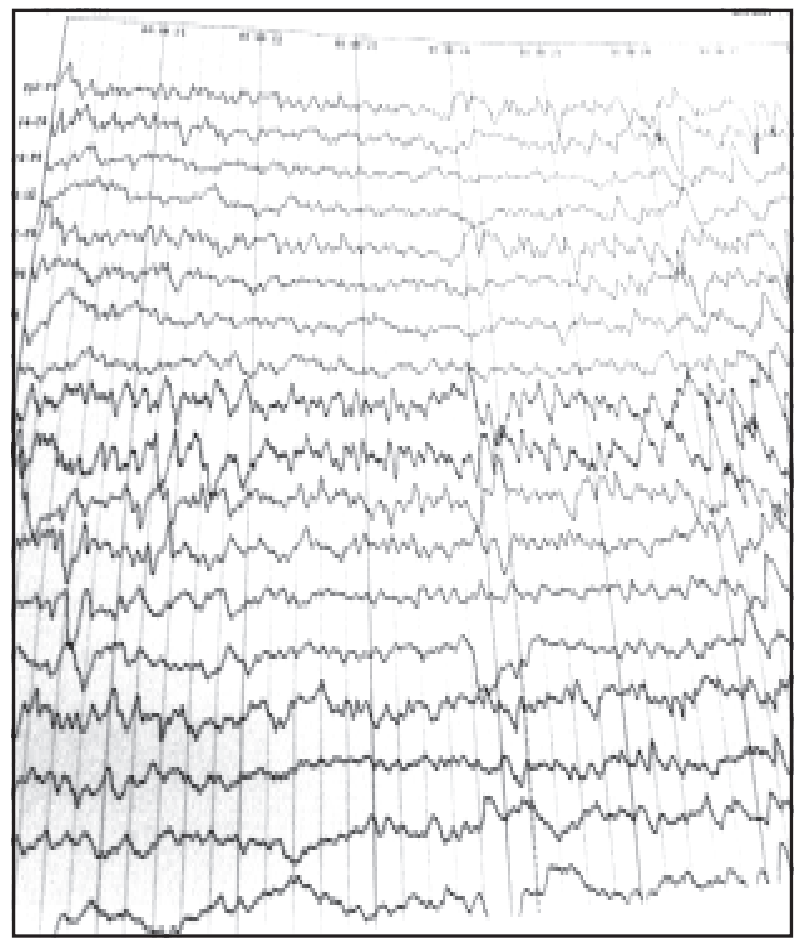

Fig.-1: EEG of the patient

\section{Discussion}

Benign neonatal sleep myoclonus (BNSM) has been described only recently. ${ }^{4}$ Coulter and Allen in 1982 described 3 infants, who had sleep myoclonus that began in the neonatal period. The myoclonic jerks were bilateral, repetitive and located mainly forearms and hands. Neurological examination and EEG were normal and remained normal during follow-up. They coined the term BNSM for this phenomenon. ${ }^{4}$ Subsequently till 1992 about 34 cases of BNSM had been described in the literature. ${ }^{2}$ It is possible that cases of BNSM are treated as neonatal seizures resulting in under diagnosis of the entity. 2,3 
International Classification of Sleep Disorders, revised: Diagnostic and Coding Manual (2nd Edition), as follows: ${ }^{5}$

- Repetitive myoclonic jerks that involve the whole body, trunk, or limbs.

- Movements that occur in early infancy, typically from birth to age 6 months.

- Movements that occur only during sleep.

- Movements that stop abruptly and consistently when the child is aroused.

- A disorder that is not better explained by another sleep disorder, by a medical or neurologic disorder, or by medication use.

The cause of benign neonatal sleep myoclonus is unknown. However, 2 reports indicate a probable genetic contribution, with several individuals affected within 2 pedigrees. ${ }^{6,7}$

Several features of BNSM help in its recognition as well as its differentiation from neonatal seizures. Though the myoclonic jerks in BNSM can be focal, multifocal, generalized or marching $1,2,8$ and mimic neonatal seizures, they characteristically appear only during sleep. ${ }^{1,9}$ More important they stop abruptly and consistently when the child is aroused. ${ }^{1,2,8}$ This latter finding needs to be elicited in all cases where a diagnosis of BNSM is considered, as it differentiates BNSM from a neonatal seizure more emphatically than its occurrence during sleep (neonatal seizures can occur during sleep). The general condition of the child is completely unaffected, even during prolonged episodes of myoclonic jerks, ${ }^{1}$ and there are no associated abnormalities on neurological or developmental examination. ${ }^{1,10}$ There is evidence that jerks of BNSM may be enhanced or precipitated by sound, touch, rocking or even benzodiazepines. ${ }^{2,4}$ True neonatal seizures, on the other hand, in most circumstances occur in the setting of perinatal, metabolic or infectious insult. ${ }^{11,12}$ Seizures do not necessarily occur during sleep, are frequently associated with change in color (cyanosis) and abnormalities on neurological and develop-mental assessment are frequently present.

Concomitant EEG abnormalities are conspicuous by their absence in BNSM. ${ }^{1,2}$ This has been confirmed by long-term video-electroencephalographic monitoring. ${ }^{9}$ How-ever, an EEG is not essential for diagnosis if attention is paid to the clinical information, but may be required to distinguish BNSM from benign neonatal convulsion ${ }^{2}$ Neonatal sleep myclonus is self limited disorder and spontaneous resolution occurs between 2 to 6 months of age. Neurological and developmental deficits do not occur on follow-up. ${ }^{1,2,8}$ BNSM is frequently misdiagnosed, there-fore, investigated and treated as a neonatal seizure, as happened initially in our cases. This reflected a lack of awareness, about the disorder. Since spontaneous resolution occurs by 6 months in almost all the patients, it may be incorrectly attributed to anticonvulsant medication and true diagnosis of BNSM never made.

BNSM should be considered as a differential diagnosis of neonatal seizures especially when seizure like activity occurs in a neurologically normal newborn. Occurrence of jerks during sleep and their disappearance on arousal should suggest a diagnosis of BNSM. Consideration of BNSM at the initial evaluation of a suspected neonatal seizure disorder, rather than making it a diagnosis of exclusion would prevent the unnecessary investigations and treatment.

The prognosis is good, and no long-term residual sleep or neurobehavioral difficulties have been identified. Parents should be reassured that their child is normal and that no long-term implications are known. However, studies with follow-up longer than 1 year remain to be completed.

\section{Conclusion}

Benign neonatal sleep myoclonus can be differentiated from epileptic jerks by myoclonic jerks that occur only while the infant is asleep or drowsy, disappear after arousal, and are associated with normal electroencephalography. Broader awareness of benign neonatal sleep myoclonus among pediatricians is important to prevent both complex and largely unnecessary testing and drug management, as well as apprehension both in the parents and in the physicians. In most cases, the diagnosis of benign neonatal sleep myoclonus is suspected on clinical grounds alone.

\section{References}

1. Blennow G. Benign infantile nocturnal myoclonus. Acta Pediatr Scand 1985; 74: 505-07.

2. Daoust-Roy J, Seshia SS. Benign neonatal sleep myoclonus. A differential diagnosis of neonatal seizures. Am J Dis Child 1992; 146: 1236-41. 
3. Reggin JD, Johnson MI. Exacerbation of benign neonatal sleep myclonus by benzo-diazepines. Am Neurol 1989; 26: 455.

4. Coulter DL, Allen RJ. Benign neonatal sleep myoclonus. Arch Neurol 1982; 32: 191-92.

5. American Academy of Sleep Medicine. International Classification of Sleep Disorders, revised: Diagnostic and Coding Manual. 2nd ed. Chicago, IL: 2001:211-2.

6. Cohen R, Shuper A, Straussberg R. Familial benign neonatal sleep myoclonus. Pediatr Neurol 2007; 36: 334-37.

7. Vaccario ML, Valenti MA, Carullo A, et al. Benign neonatal sleep myoclonus: case report and follow-up of four members of an affected family. Clin Electroencephalogr. Jan 2003; 34: 15-17.

8. Resnick TJ, Moshe SL, Perotta L, Chambers HJ. Benign neonatal sleep myoclonus. Relation to sleep states. Arch Neurol 1986; 43: 266-68.

9. Di Capua M., Fusco L, Ricci S, Vigevano F. Benign neonatal sleep myoclonus: Clinical features and video-polygraphic recordings. Mov Disord 1993; 8:191-94.

10. Alfonso I, Apazian O, Jeffries HE. A simple maneuver to provoke benign neonatal sleep myoclonus. Pediatrics 1995; 96: 1161-63.

11. Volpe JJ. Neonatal seizures. In: Neurology of the Newborn. Ed. Philadelphia, WB. Saunders Co, 1995; pp 172-207.

12. Evans D, Levene M. Neonatal seizures. Arch Dis Child 1998; 78: 70-75. 